\title{
Cost of a WARC Analyzing Web Archives in the Cloud
}

\section{Ryan Deschamps, Samantha Fritz, Jimmy Lin, Ian}

\section{Milligan, and Nick Ruest}




\section{Why do we care about web archives?}

Born-digital sources have the potential to reshape research in the humanities and social sciences;

Research access has lagged (beyond Wayback Machine, analysis ecosystem is mostly command-line-based tools)

As we plan for research access, we need to understand the economics associated with providing this sort of access

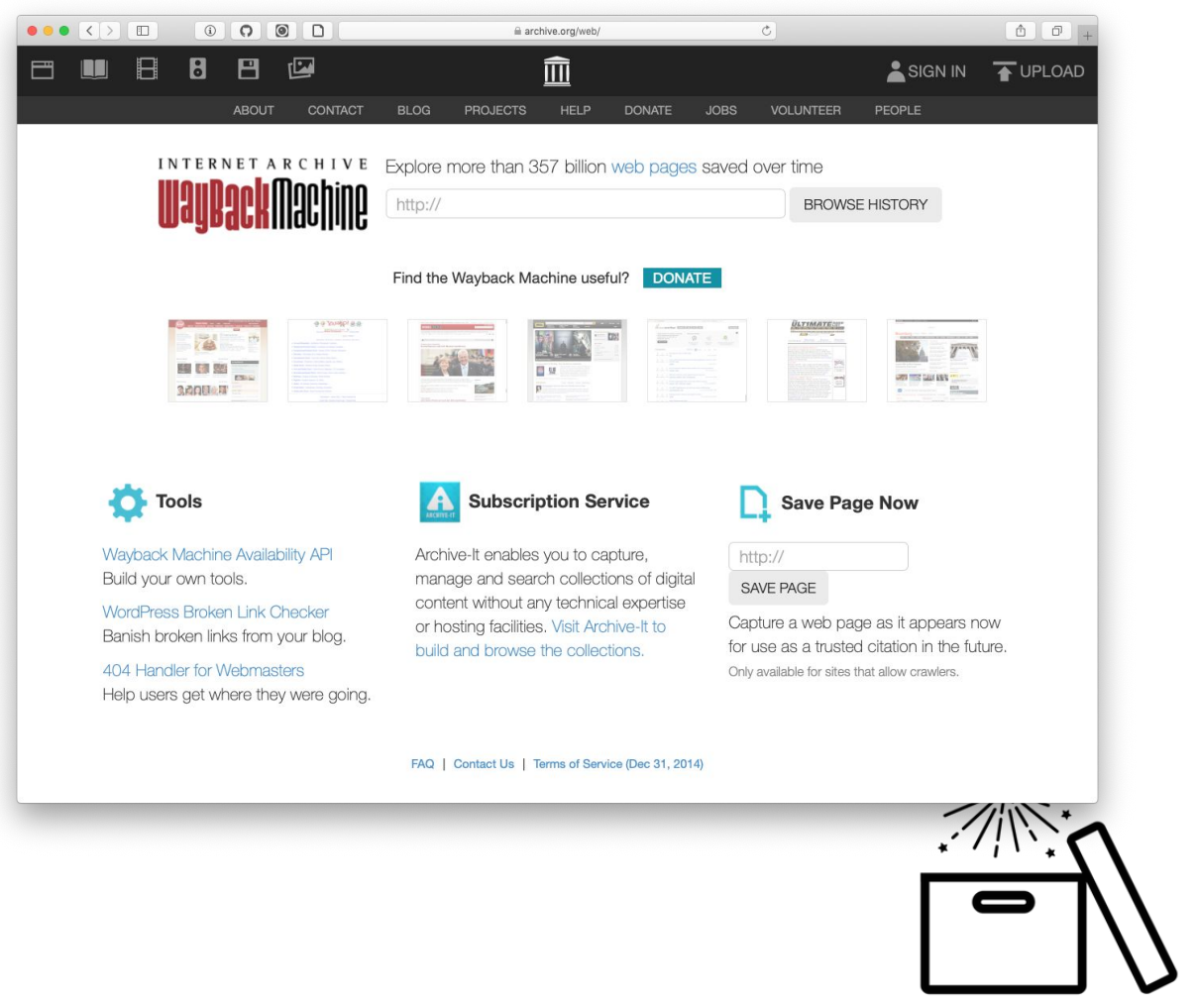




\section{How much does it cost}

to analyze a WARC (the standard container file format of web archives) in the cloud?

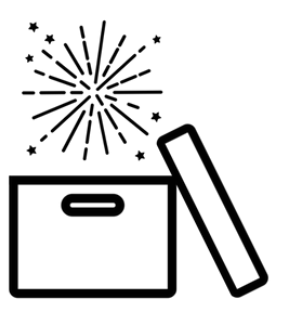



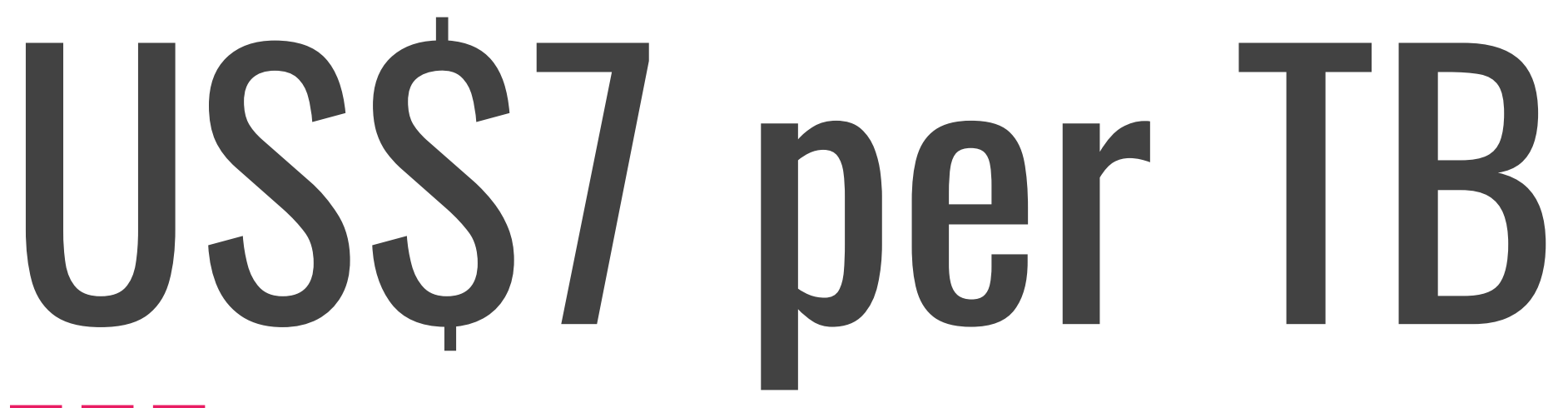

The TL;DR

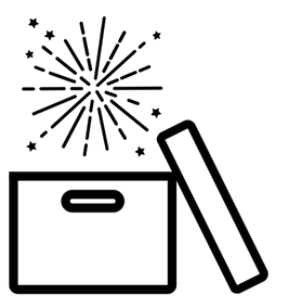




\section{What do we mean by the "Cloud"?}

We conduct our work on the Compute Canada Cloud, which is an OpenStack instance supported by a research grant.

As OpenStack is a popular open-source cloud platform, our findings should be generalizable.

We translated all of our compute time into Amazon Web Services costs as it is the most popular commercial provider.

compute calcul

canada canada

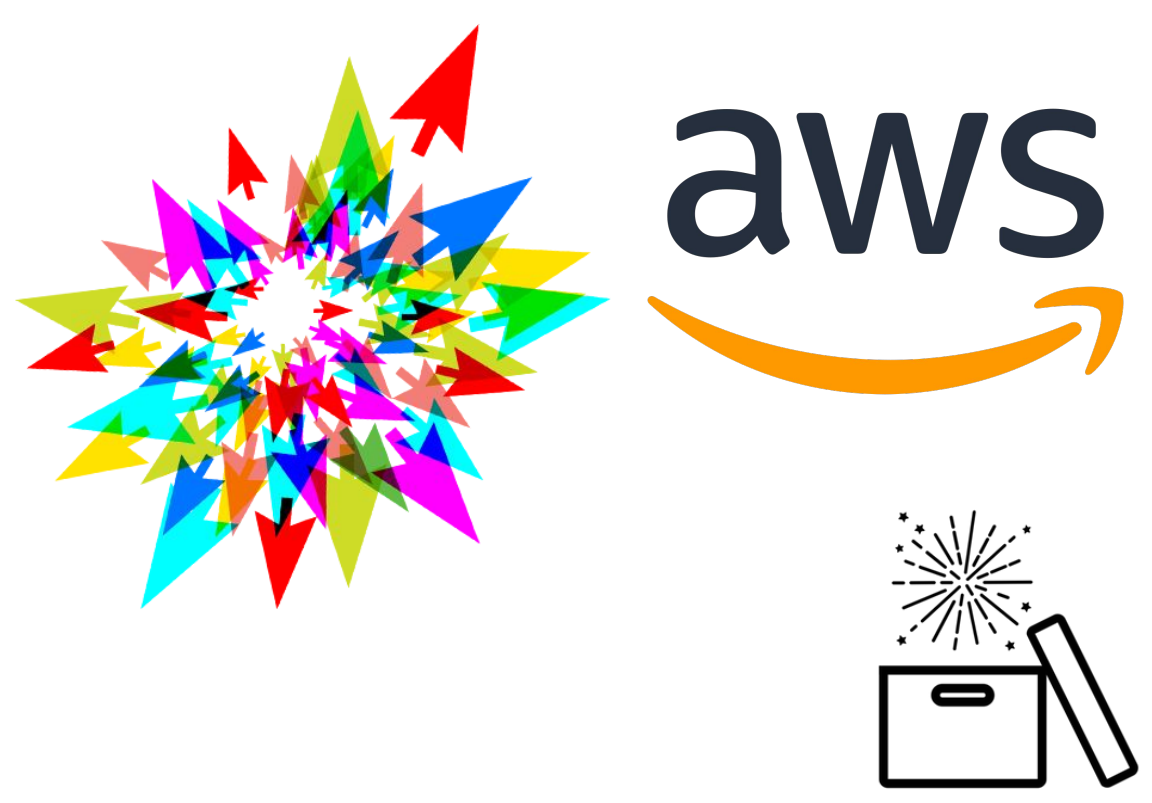




\section{What are we performing "analysis" with?}

Analysis using the Archives Unleashed Toolkit or AUT

AUT is a Scala domain-specific language on top of the Apache Spark

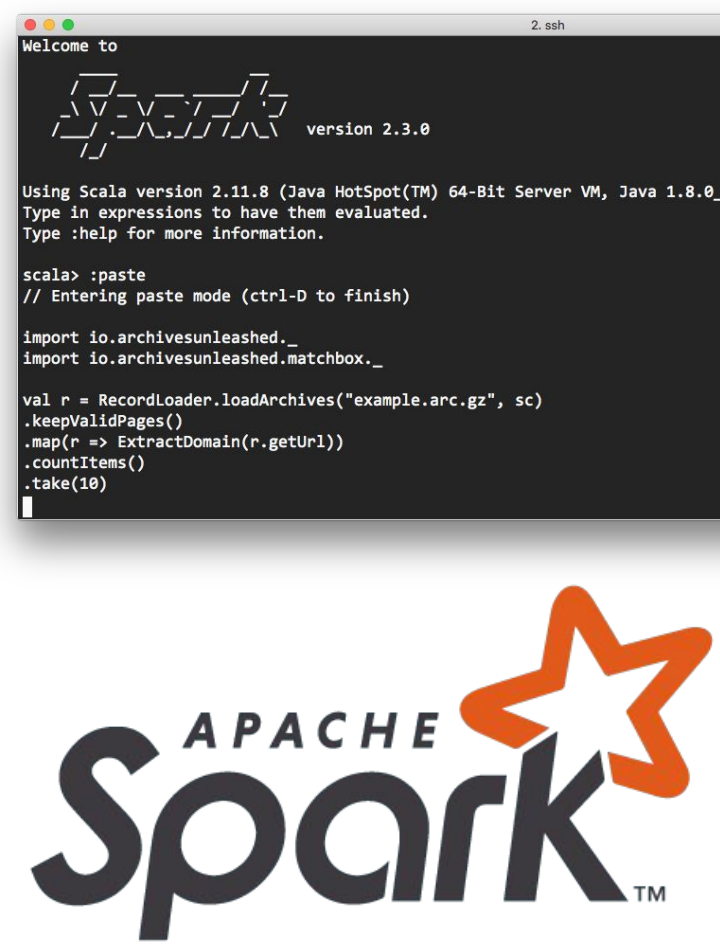
platform

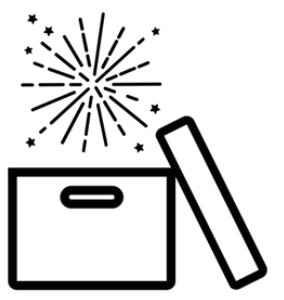




\section{What do we mean by "Analysis"?}

The Filter - Analyze - Aggregate Visualize (FAAV) Cycle

Common analytics task: crawl statistics to visualizing web graphs to exploring text at scale

Informed by extensive hands-on collaboration

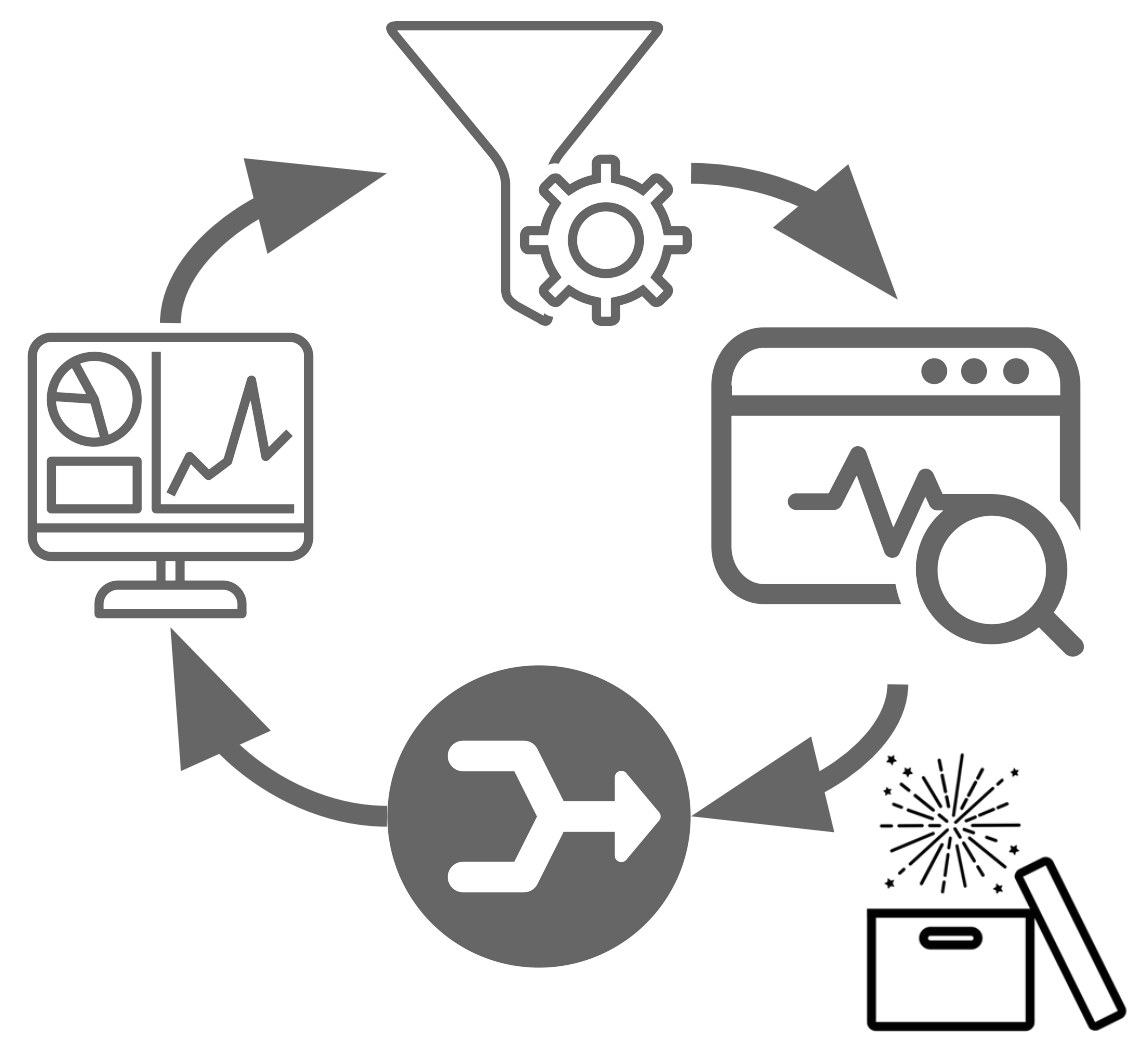



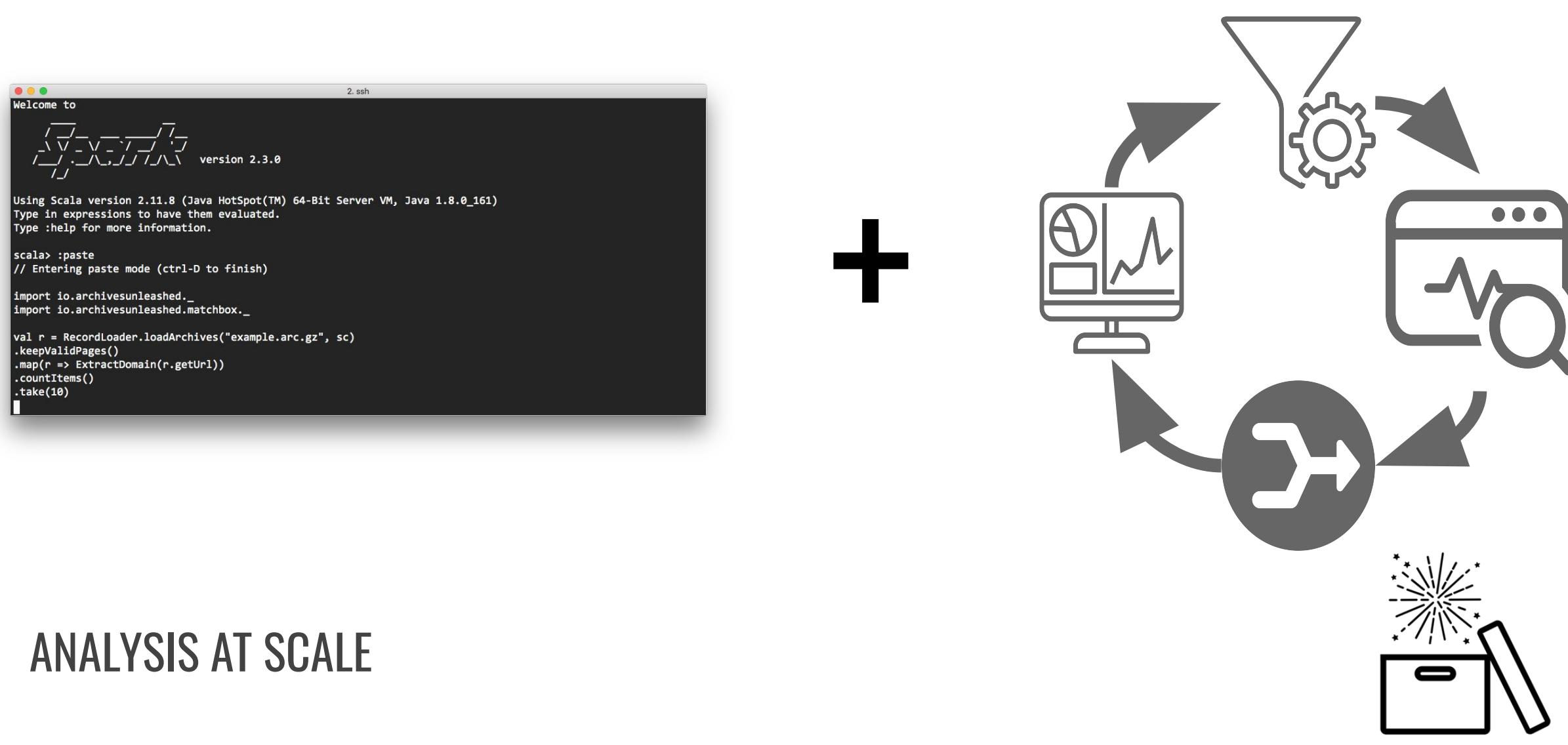

ANALYSIS AT SCALE 


\section{What do we mean by "Analysis"?}

Extract all URLs to compute the frequency of domains appearing in a given collection (domain distribution);

Extract all plain text from all pages, along with metadata such as crawl date, domain name, and URL (full text); and

Extract all hyperlinks to create a domain-to-domain network graph (webgraph);
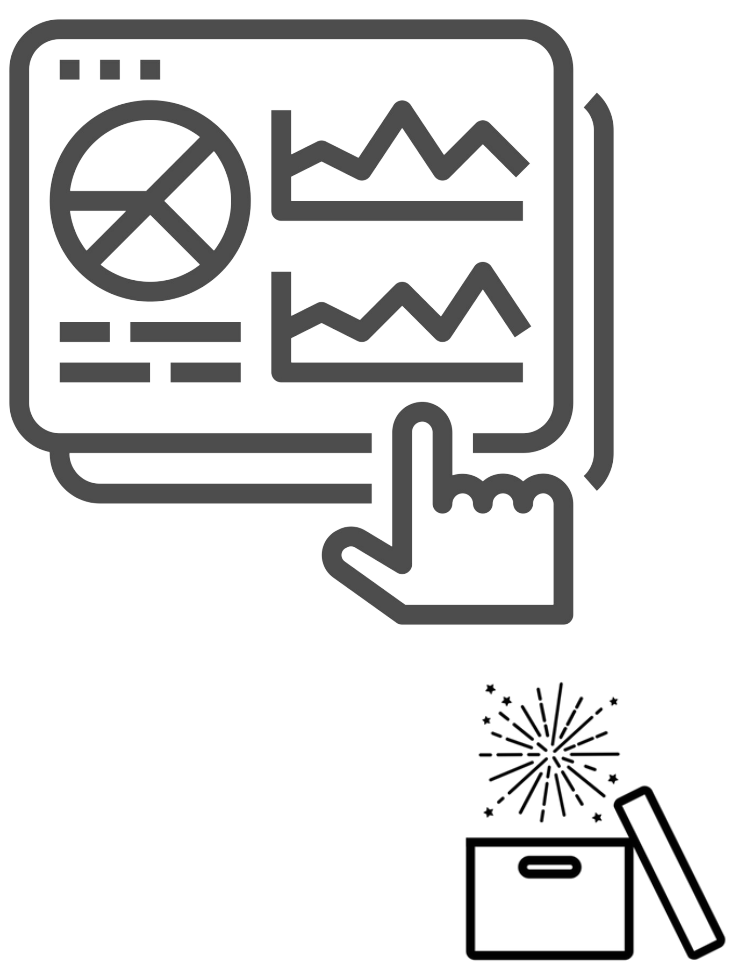
On to the experiment!

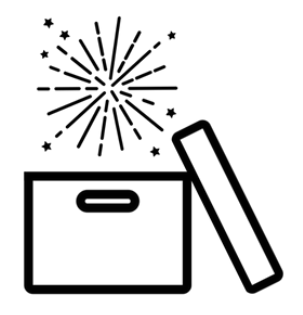




\section{The Experiment}

We decided to use a 16 core, $64 \mathrm{~GB}$ memory virtual machine

Powerful, but struck the balance between expensive and power

Why not a cluster?

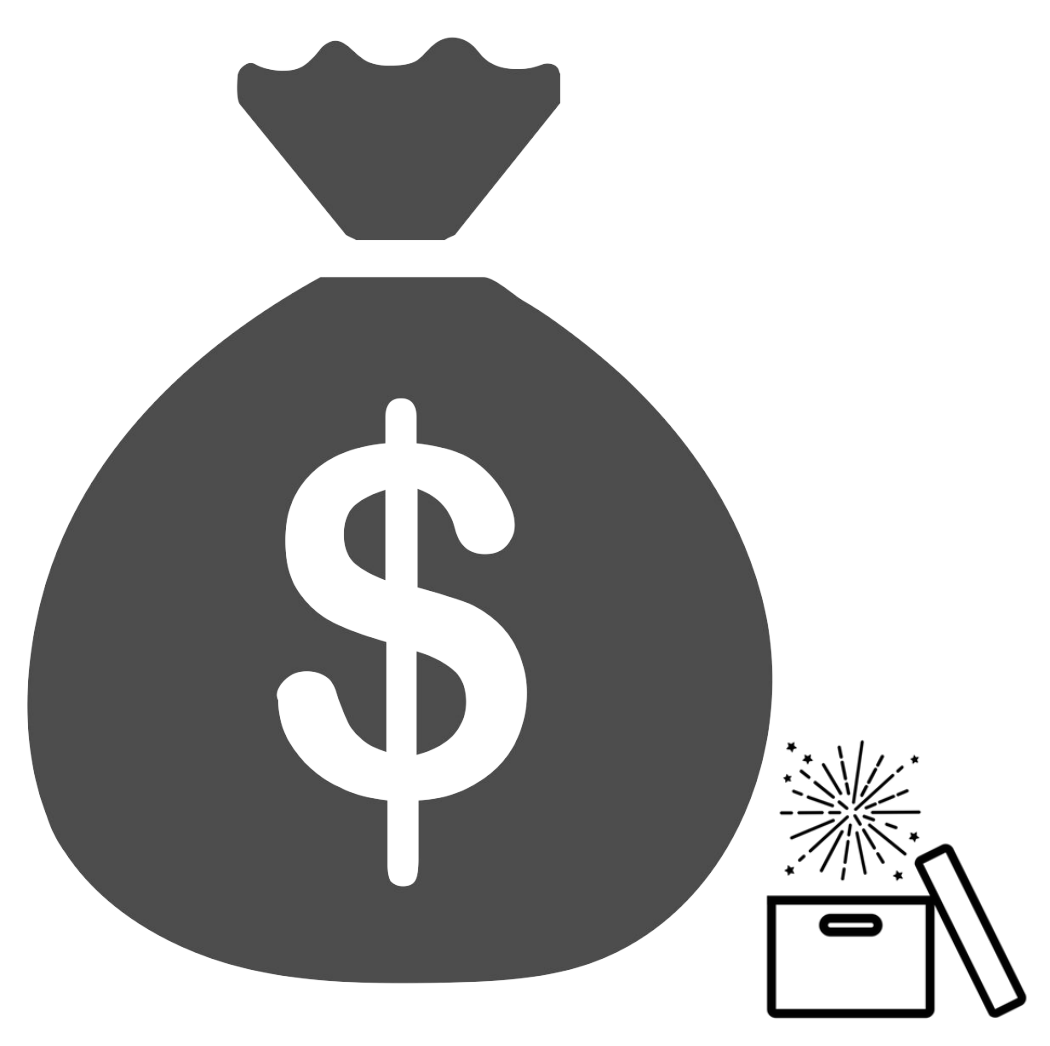




\section{The Experiment}

Analysis based on analyzing the cost of processing $\mathbf{4 8}$ Archive-It collections from six Canadian universities (Toronto, Victoria, Simon Fraser University, Manitoba, Dalhousie, and Winnipeg).

\begin{tabular}{lr}
\hline Size & Count \\
\hline$\geq 1 \mathrm{~GB},<10 \mathrm{~GB}$ & 10 \\
$\geq 10 \mathrm{~GB},<100 \mathrm{~GB}$ & 18 \\
$\geq 100 \mathrm{~GB},<1 \mathrm{~TB}$ & 15 \\
$\geq 1 \mathrm{~TB}$ & 5 \\
\hline Total & 48 \\
\hline &
\end{tabular}

A variety of sizes - smallest at 1.2GB was Victoria's academic calendar; largest at 4.3TB was Canadian Government Information Collection 


\section{The Experiment (Workflow)}
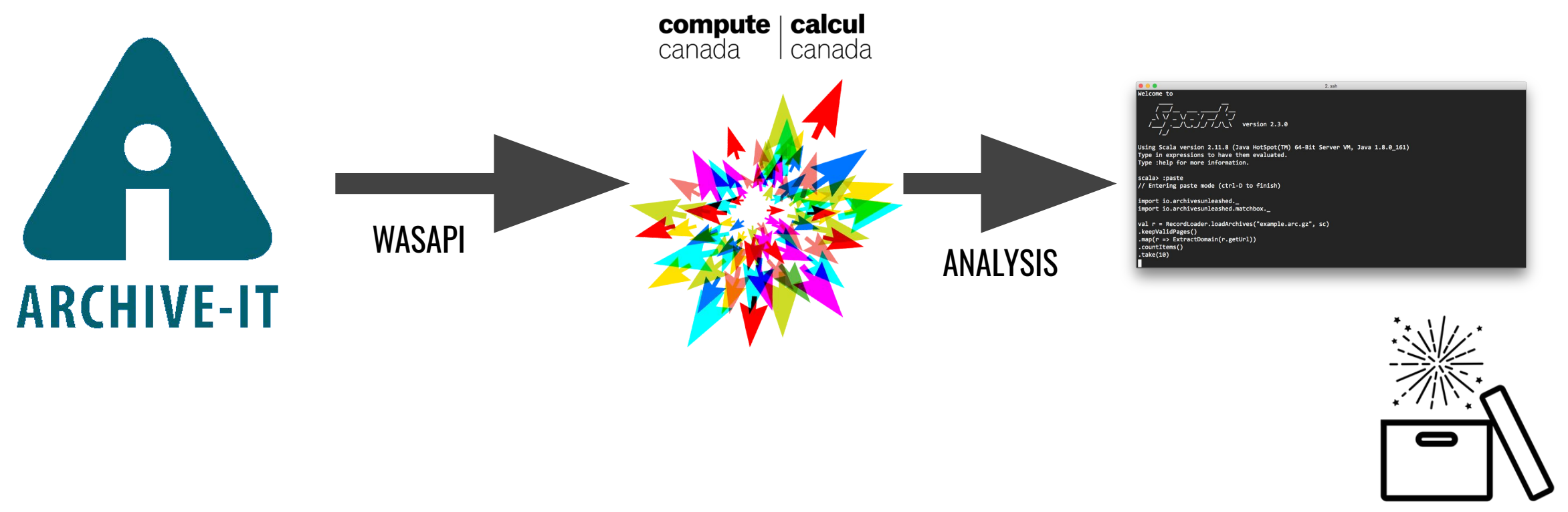


\section{Findings}

We then took all the times for each job (Domain, Full Text, Webgraph) and found processing time per GB in seconds.

\begin{tabular}{lrrrr}
\hline Derivative & all & L & M & S \\
\hline domain distribution & 32 & 25 & 27 & 36 \\
full text & 34 & 28 & 35 & 34 \\
webgraph & 36 & 34 & 36 & 36 \\
\hline total & 102 & 87 & 98 & 106 \\
\hline
\end{tabular}

Processing times drop as size increases, as startup costs are

Figure: Processing times per GB in seconds amortized.

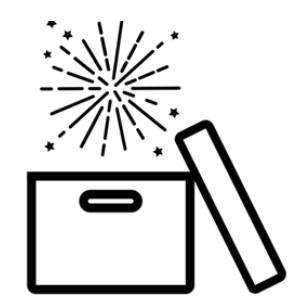




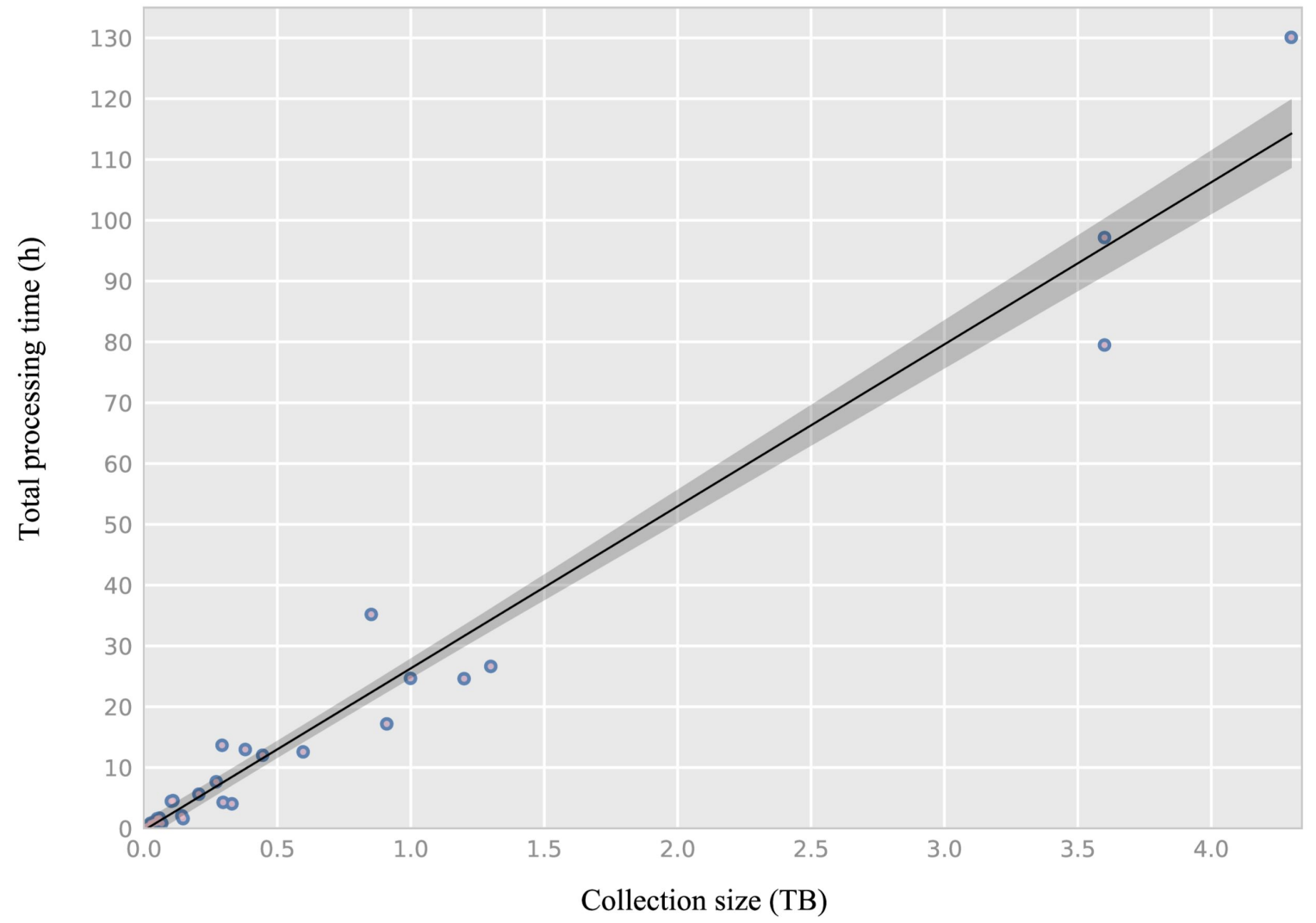

Scatter plot between collection size and total processing time, illustrating a linear relationship

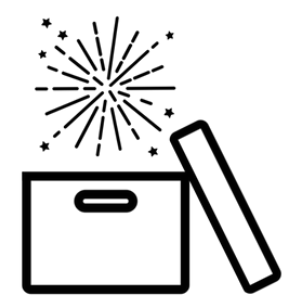




\section{Findings}

Derivative files are much smaller

Researcher can usually work with these derivative files on their own systems in a way they could not

\begin{tabular}{lrrrr}
\hline Derivative & all & L & M & S \\
\hline domain distribution $(\mathrm{KB})$ & 0.95 & 0.51 & 0.98 & 1.01 \\
full text $(\mathrm{MB})$ & 78.5 & 97.6 & 102.1 & 62.4 \\
webgraph $(\mathrm{KB})$ & 76.9 & 85.8 & 122.6 & 50.9 \\
\hline
\end{tabular}

Figure: Derivative sizes per GB work with their WARCs

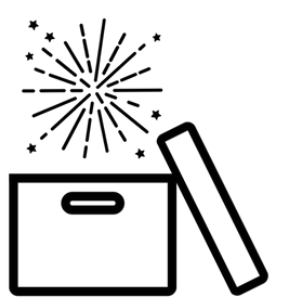




\section{So we know the times to compute these derivatives.. Show me the money!}

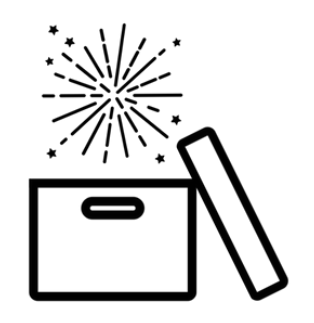




\section{Derivative}

all

L

M

S

\begin{tabular}{lllll} 
domain distribution & $\$ 6.51$ & $\$ 4.67$ & $\$ 5.05$ & $\$ 7.63$ \\
full text & $\$ 6.73$ & $\$ 5.24$ & $\$ 6.65$ & $\$ 7.04$ \\
webgraph & $\$ 7.19$ & $\$ 6.46$ & $\$ 6.82$ & $\$ 7.52$ \\
\hline
\end{tabular}

total

$\$ 20.43$

$\$ 16.37$

$\$ 18.52$

$\$ 22.19$

Processing cost per TB in US $\$$

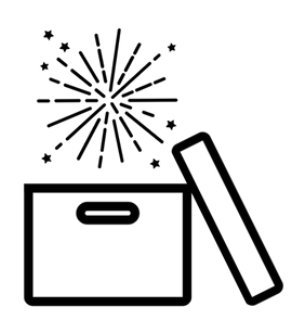




\section{Cost of a WARC}

C5.4xlarge (16 core, 68 GB memory) is $\$ 0.68 /$ hour in US East (Ohio)

The previous results show a macro-average

The bottom line: US\$7/TB for a typical analytics operation such as generating domain frequency reports, extracting full text of a collection, or extracting the link-to-link webgraph of hyperlinks.

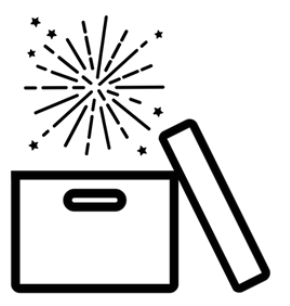




\section{Cost of a WARC}

\section{This is cost-competitive}

Google BigQuery costs USS5 per TB - but is SQL based and prices on uncompressed size whereas our calculations were on compressed WARCs (which are roughly $60 \%$ the size of uncompressed WARCs)

Archives Unleashed is price competitive with commercial services, albeit without any profit margin.

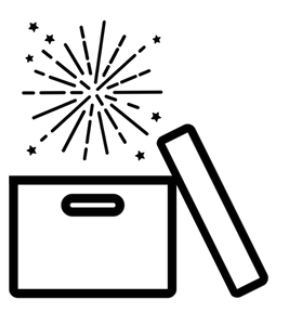




\section{Proposed Workflow}

Cheaper download server (ex. t3.medium)

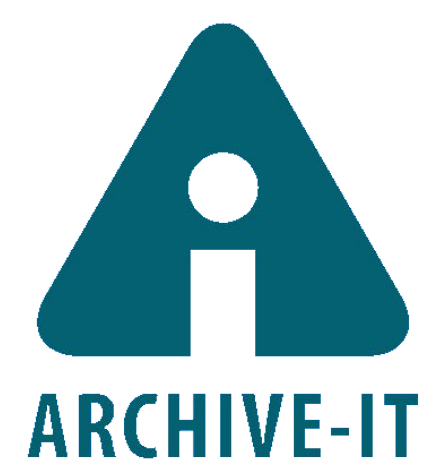

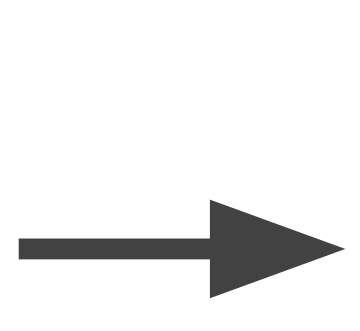

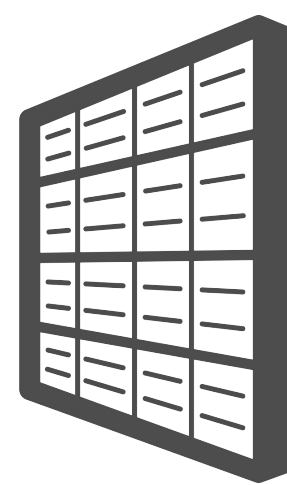

Expensive processing server (ex. c5.4xlarge)

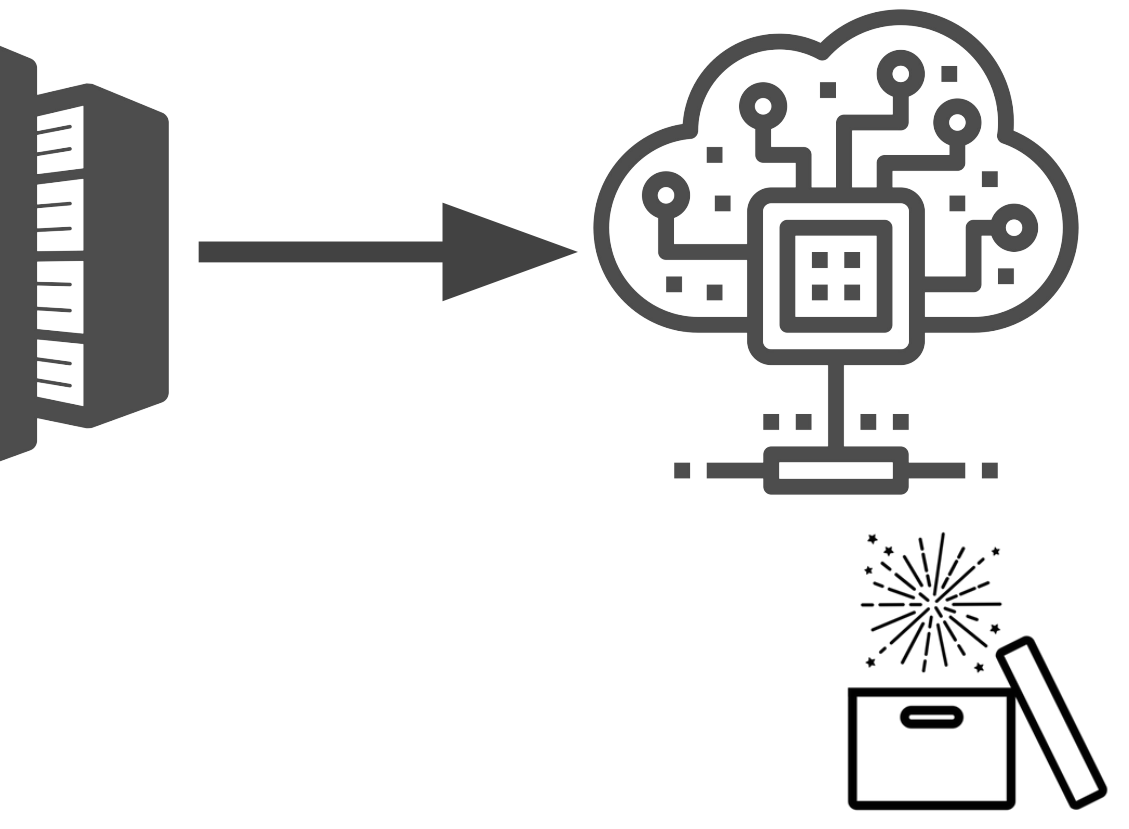




\section{Limitations: Storage}

We did not include storage in this discussion. 1TB of data costs US\$23 per month. Our preferred workflow would be to transfer WARCs, analyze, and then delete them quickly.

At $30 \mathrm{MB} / \mathrm{s}$ data transfer speed, transferring a TB costs US\$0.40; less than the per-day cost of $\$ 3$ data storage

As long as the preservation copy is secure, the "processing copy" can be created and deleted on a whim
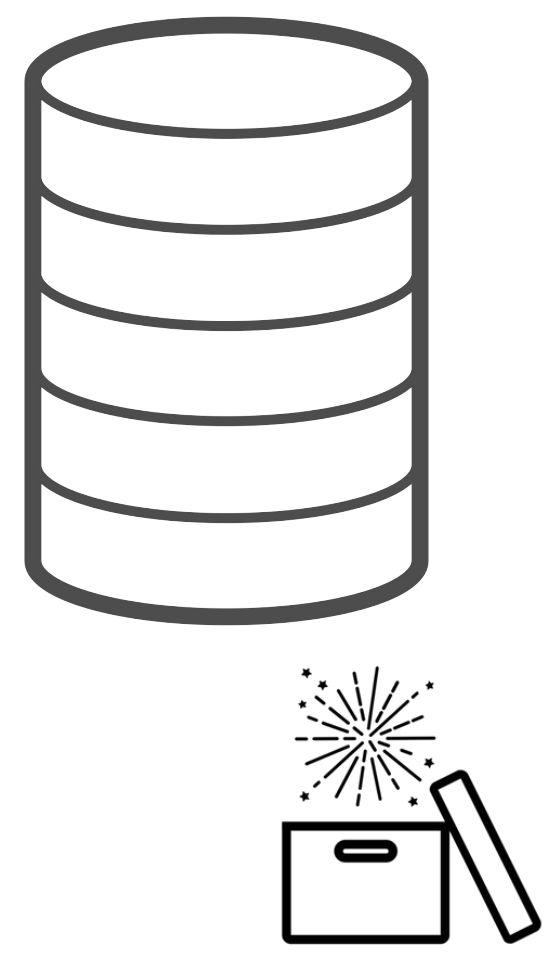


\section{Conclusions}

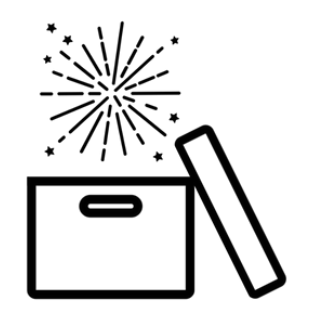




\section{Conclusions}

Archives Unleashed Cloud

Archives Unleashed C $>\equiv$

\section{Nova Scotia Municipal Governments}

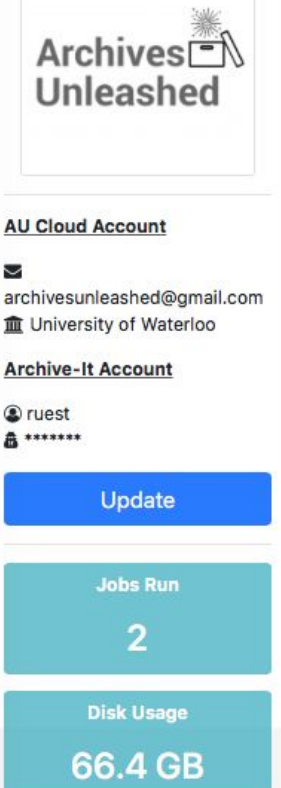

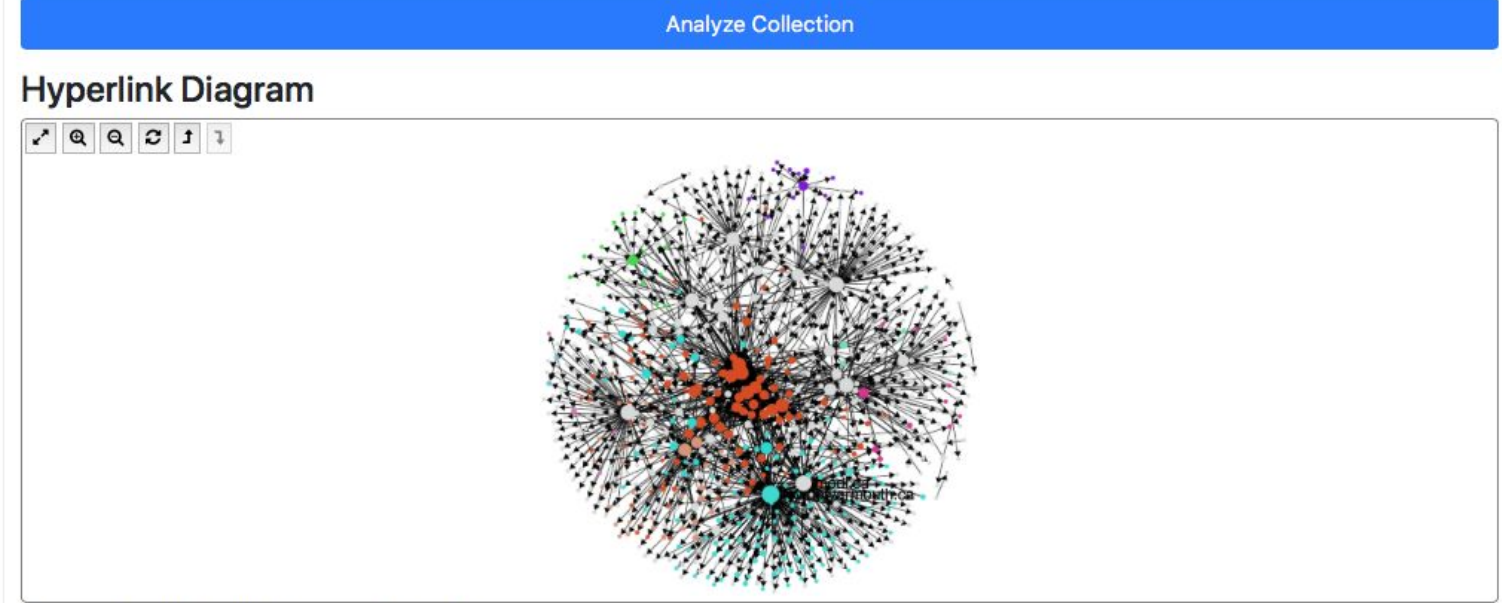

Download Collection Derivatives

$$
\begin{aligned}
& \text { Gephi } \\
& \text { Yow Network } \\
& \text { You can find information about how to use these files here. }
\end{aligned}
$$


We share the beginnings of an economic analysis and

believe the costs to be quite

affordable; whether

institutions or individual

scholars find these costs

palatable remains to be seen.

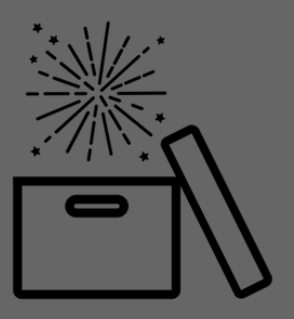



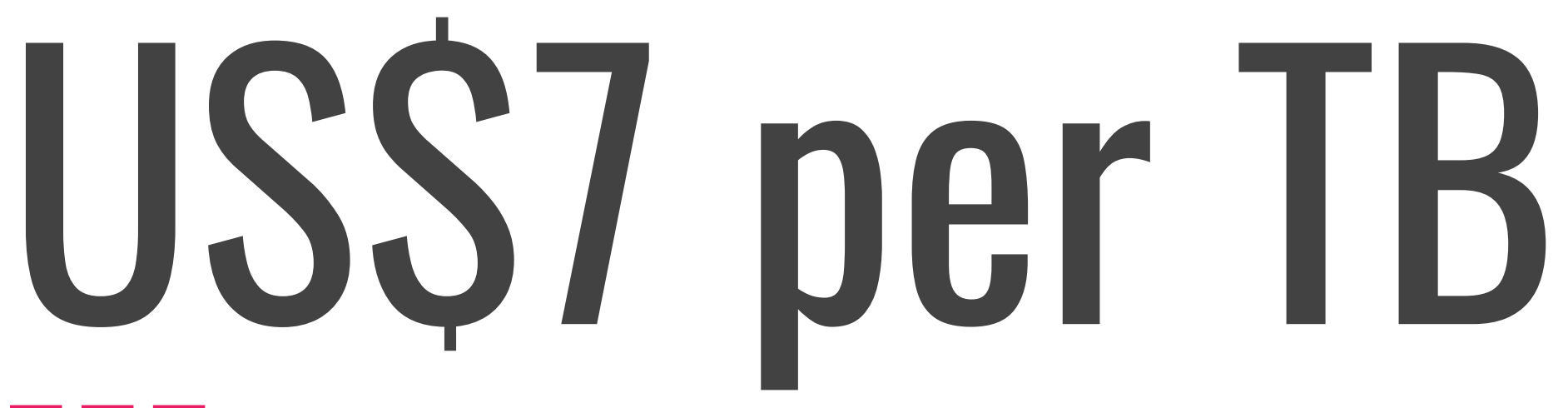

The TL;DR

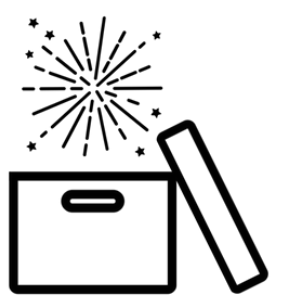




\section{Thanks to our supporters!}

THE

ANDREW W.

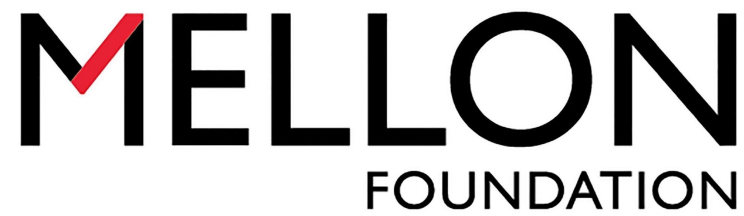

compute calcul

canada canada

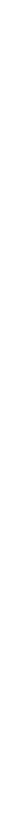

